\title{
Classical eyelid conditioning of the severely retarded: Partial reinforcement effects'
}

\author{
Leonard E. Ross, Charles H. Koski and Judy Yaeger \\ UNIVERSITY OF WISCONSIN
}

\begin{abstract}
Abstraet
Eyelid conditioning data was obtained from severely retarded Ss by using a motion picture as a distractor during the experimental session. In contrast to findings with normal Ss, there was little reinforcement decrement in acquisition, and partial reinforcement Ss extinguished as rapidly as those receiving continuous reinforcement.

Problem
\end{abstract}

Eyelid conditioning with humans has been almost entirely limited to the use of college students as Ss, despite the potential usefulness of classical conditioning data obtained from retardates or normal children. This paper reports the use of a procedure intended to resolve the difficult problem of controlling the retardate or child in the experimental situation. Severly retarded Ss were shown a motion picture during conditioning and extinction periods, a procedure designed to distract the Ss so they would tolerate the situation and leave the headset undisturbed. Continuous and partial reinforcement groups were run since the commonly reported effects of partial reinforcement in eyelid conditioning, i.e. a decrement in acquisition and greater resistance to extinction, are presumedly due to inhibitory factors, and retardates supposedly suffer from inhibitory deficits relative to normals. College students were also run under motion picture conditions to control for the possibility that this procedural feature might affect continuous-partial differences.

\section{Method}

Retarded Ss were selected from wards for the severely retarded at two institutions. Mean MA's were 34.2 and 35.0 months, chronological ages 17.2 and 23.6 years, and IQ's 18.0 and 19.9 for the $100 \%$ and $50 \%$ groups respectively. The MA and IQ scores were obtained from Peabody Picture Vocabulary Tests. Normal Ss were 48 students from an Introductory Psychology class at Peabody College.

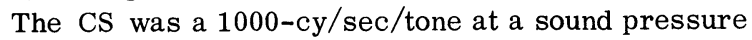
level of $85 \mathrm{db}$ (relative to $2 \times 10-4$ dyne $/ \mathrm{cm}^{2}$ ). The CS duration was $550 \mathrm{msec}$., CS-UCS interval was $500 \mathrm{msec}$, and the UCS was a $1.0 \mathrm{1b} / \mathrm{in}^{2}$ air puff of $50 \mathrm{msec}$. duration. Intertrial interval varied from 15 to $25 \mathrm{sec}$. , with a mean value of $20 \mathrm{sec}$. Two films were used with movie groups, one showing animals in wildlife settings, the other depicting the life of children in various cultures. The projector was located in the room with the subject. Any response $1 \mathrm{~mm}$ or more in amplitude occuring 200 msec. following the onset of the CS, but before the unconditioned response latency, was scored as a condi- tioned response. An experimenter who initiated the trials sat next to retarded Ss to insure that the apparatus was undisturbed. Fifty acquisition trials were given on each of two consecutive days, while 10 acquisition trials were immediately followed by 40 extinction trials on day three. No air puff was delivered on nonreinforced trials in acquisition or extinction. One group of retardates $(N=20)$ received $100 \%$ reinforcement during acquisition, while the other $(\mathrm{N}=20)$ received $50 \%$ reinforcement during this period. A movie was shown during all retardate sessions. Two groups of college students were run under conditions similar to those of the retardate groups, while two additional student groups received the same experimental treatment except that they were told they would not see a motion picture and the picture was replaced by a white spot of light. There were $12 \mathrm{Ss}$ in each normal group. Each retardate and normal group had an equal number of males and females.

\section{Results}

The motion picture distraction procedure was quite successful. Of the 43 retarded Ss taken to the experimental room, 40 completed the three sessions. One subject was too hyperactive to continue in the conditioning situation, one refused to sit in the chair, and one would not remain in the chair once seated. Figure 1 presents the acquisition and extinction curves for the retardate and normal groups. The $100 \%-50 \%$ differences for retardates on day 1 , day 2 , trial block 11 , and during extinction all failed to reach the .20 level (t tests), while these differences for the normals were significant at the $.001, .005$, and .01 levels respectively when tested by analysis of variance also involving the movie-no movie factor. There were no significant movie-no movie effects or interactions. The number of conditioned responses made by each subject on the last block of 10 acquisition trials was compared with performance on the first block of 10 extinction trials. These difference scores for the $100 \%$ and $50 \%$ groups were significantly different in the case of retardates $(p<.02)$ and for both movie $(p<.01)$ and no-movie $(p<.01)$ conditions in the case of normals. However the normal-retardate effects were in opposite directions, the acquisition to extinction drop being greater in the case of the $50 \%$ retardate group and the $100 \%$ normal groups. Examination of the graphs shows similar rates of extinction for the retardate reinforcement groups, but the superior resistance to extinction of the partial normal group is evident, if allowance is made for the final conditioning level. The movie-no movie effect for normals in extinction reached the .10 but not the .05 level, with movie Ss at higher levels. 

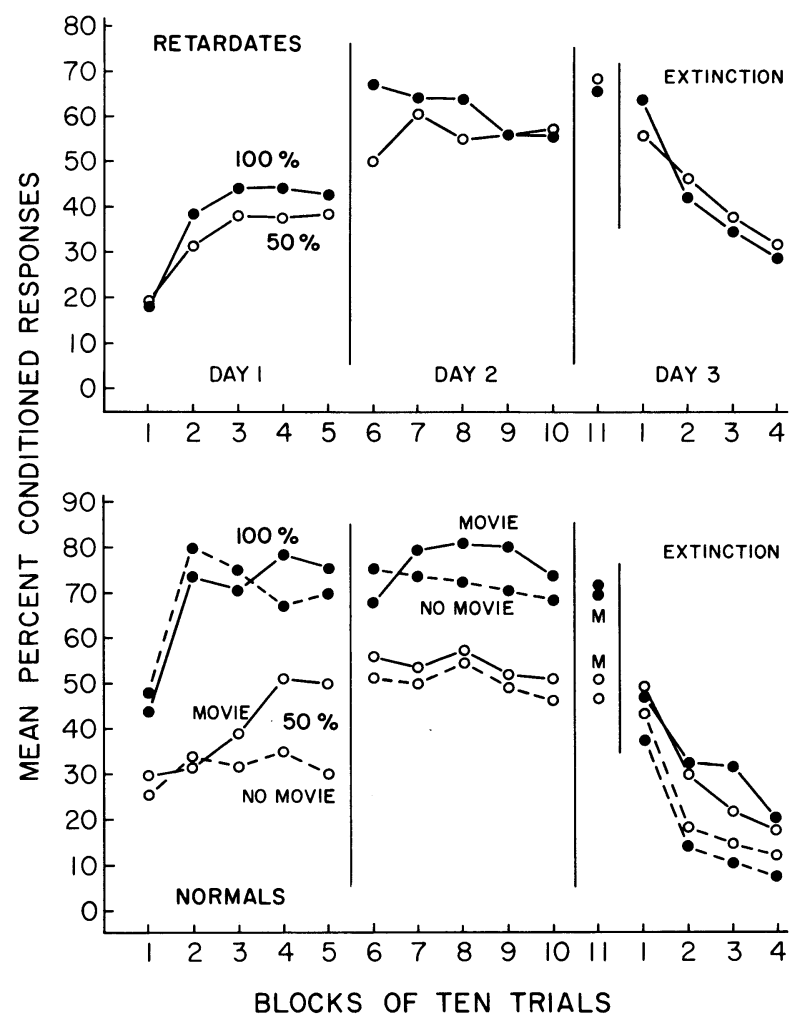

Fig. 1. Percentage of conditioned responses made by normal and retarded subjects as a function of reinforcement schedule.

A large number of responses made by the $100 \%$ retardate group were of the "voluntary" type by a slope of response criterion (Hartman \& Ross, 1961). However the majority of these responses did not have the other form characteristics of voluntary responses, since the eye opened again and remained open until the UCS was received. Criteria currently used to detect "voluntary" responding are apparently inappropriate for this population. Three Ss in the 50\% normal groups, none in the $100 \%$ normal groups, were voluntary by the slope criterion.

\section{Discussion}

The relative ease with which it was possible to condition severely retarded subjects using the motion picture distraction procedure indicates that it is quite feasible to use classical eyelid conditioning with the retarded. Moreover the present techniques should also be useful with normal children, since the procedural problems involved in work with this population would appear to be similar to those encountered with retardates.

This study demonstrates that the effects of partial reinforcement in the acquisition and extinction of the conditioned eyelid response are quite different for retardates and college students, and raises the possibility that the inhibitory, or inhibitory set, factors involved are less operative with retardates. However it is not possible to determine from the present data whether these contrasting effects are related to MA or IQ differences.

\section{heference}

HARTMAN, T. F., \& ROSS, L. E. An alternative criterion for the elimination of "voluntary" responses in eyelid conditioning. J.exp. Psychol., 1961, 61, 334-338* Note

1. This research was supported by National Science Foundation grant GB-765. The first author was a Joseph P. Kennedy Foundation Fellow at Peabody College, and the second author was a USPHS Predoctoral Fellow, during the major part of the study. The authors are indebted to Central Wisconsin Colony, Madison, and Cloverbottom Hospital and School, Donelson, Tennessee for their cooperation in providing facilities and subjects. 